\title{
Responsabilidad social empresarial Necesidad y creatividad
}

\author{
Fecha de recepción: 9 de agosto de 2010 - Aprobación: 13 de septiembre de 2010
}

\section{Álvaro Enrique Rodríguez Hernández}

\section{Resumen}

Este artículo presenta la creatividad como un punto de vital importancia en el momento de pensar en la responsabilidad social empresarial (RSE) de las organizaciones. Determina y sustenta la necesidad que tiene el país de contar con empresas comprometidas con este tipo de procesos de RSE. Muestra casos exitosos de RSE y consulta fuentes directas, que conceptualizan y ofrecen un marco contextual del desarrollo y fundamentos de la RSE. Aporta una entrevista con uno de los más destacados estudiosos de la responsabilidad social empresarial de España, en la cual se puede conocer la situación en este campo en Europa y quien da unas recomendaciones para optimizar la implementación de la RSE.

\section{Abstract}

The paper presents creativity as a point of vital importance when thinking about the corporate social respon- sibility of organizations. It determines and supports the need of the country to have companies committed to CSR processes. It also shows successful cases of CSR and consults direct sources that conceptualize and provide a contextual framework for the development and fundamentals of CSR. The article presents an interview with one of the leading scholars of corporate social responsibility in Spain, in which it is possible to know the status of this field in Europe and to have some recommendations in order to optimize the implementation of CSR.

\section{Palabras claves}

Responsabilidad social empresarial, creatividad, organizaciones, oportunidades de negocio, compromiso social.

\section{Keywords}

Corporate Social Responsibility, creativity, organizations, business opportunities, social commitment.
José Miguel Insulza, secretario general de la Organización de Estados Americanos (OEA), afirmó: "La pobreza y la inequidad repercuten en la calidad de la democracia e impiden su pleno ejercicio en la región", asimismo dijo: "Que más de un tercio de los habitantes de la región siga viviendo en condición de pobreza no corresponde a un continente con nuestro nivel de desarrollo". Según cifras del DANE el 48,3\% de hogares a escala nacional se consideran pobres.

Luis Pérez Gutiérrez, en el periódico $E l$ Mundo de Medellín, sostuvo: "En América Latina hay 34 millones de analfabetas abso- 
lutos, lo cual envía una señal de que tan grave mal convive con la posmodernidad". Radio Múnera revela cifras de la Unesco:

Crecen las cifras de analfabetismo en Colombia. Siete de cada cien colombianos mayores de 15 años no saben leer ni escribir, pese a los esfuerzos recientes por contrarrestar este índice, aseguró la Unesco.

La capacidad de respuesta que tiene una empresa o una entidad, frente a los efectose implicaciones de sus acciones sobre los diferentes gruposcon losque se relaciona (stakeholders o grupos de interés).

Por su parte El Universal titula: "Afrocolombianos rechazan la invisibilización de sus derechos", y la Corporación Humanas Colombia publica:

El 77\% de las colombianas se sienten discriminadas y el $84 \%$ cree que Colombia es un país machista. Así lo revela la primera encuesta nacional de percepción de las mujeres sobre su situación y condiciones de vida en Colombia.

Los anteriores datos marcan un contexto que tiene cifras y casos mucho más escalofriantes que los aquí expuestos: abusos, maltratos, explotación laboral y miseria, entre otros, es una realidad a la cual no se le puede dar la espalda, pero sí se le puede dar la mano, los pensamientos utópicos y filantrópicos tienen gran cabida en la actualidad y más aún cuando se ven titulares como el de El Espectador, del 15 de febrero de 2010: "Ganancias del sector financiero llegaron a 8,5 billones de pesos".

El sector privado, además de los planes y políticas de gobierno, está llamado a dar esa

\section{Reseña de autor}

Álvaro Enrique Rodríguez Hernández

Politécnico Grancolombiano

aerodriguez@poli.edu.co

Profesional en Comunicación Social-Periodismo, especialista en Docencia Universitaria, docente de tiempo completo del Departamento Académico de Comunicación de la Facultad de Mercadeo, Comunicación y Artes del Politécnico Grancolombiano. Director del programa "La bitácora", emitido por la emisora por internet "Poliradio", columnista del periódico Golpe de Opinión y administrador del blog: mediosyperiodismo.blogspot.com mano a todos aquellos que se encuentran en condiciones desfavorables y, claro está, el ejemplo comienza por casa, una empresa con sanas costumbres laborales y un alto compromiso con el bienestar de sus colaboradores hace que esos planes se irradien a su entorno. Es decir, es necesario que cada empresa: familiares, pymes, multinacionales y demás, entren en forma programática y sostenible en el desarrollo de políticas y planes de responsabilidad social empresarial (RSE).

“¡Ay! de nuevo el mismo cuento, pero si más de una vez le hemos echado números al asunto y la plata apenas alcanza para pagar la nómina”, precisamente, desde la misma nómina se puede generar un programa de RSE. Sus colaboradores pueden ser el principio de un plan de alto impacto que incida favorablemente en el desarrollo y bienestar de una población en condiciones adversas.

Pero ¿qué es la RSE? Según el Centro Colombiano de Responsabilidad Empresarial (CCRE) es definida como:

la capacidad de respuesta que tiene una empresa o una entidad, frente a los efectos e implicaciones de sus acciones sobre los diferentes grupos con los que se relaciona (stakeholders o grupos de interés). De esta forma las empresas son socialmente responsables cuando las actividades que realiza se orientan a la satisfacción de las necesidades y expectativas de sus miembros, de la sociedad y de quienes se benefician de su actividad comercial, así como también, al cuidado y preservación del entorno.

Si la anterior definición contara con una aplicación efectiva; definitivamente, el panorama mostraría una sociedad construida desde la equidad y la igualdad de oportunidades; pero, a veces las cosas son mal interpretadas o mal manejadas, como 
bien lo explica el periodista y analista económico Francisco Leal Mateus:

La RSE se convirtió en un saludo a la bandera. La mayoría de empresas hacen donaciones a fundaciones o ONG para ayudar a las poblaciones más vulnerables, pero muchas de esas organizaciones son montadas por ellos mismos. Las ayudas de alguna $\mathrm{u}$ otra forma son descontadas de los impuestos pero retornan a la misma caja o bolsillo de los dueños.

Definitivamente, el sector privado responde a un modelo empresarial denominado y conocido ampliamente como empresa con ánimo de lucro. La RSE no es otra oportunidad para aumentar ingresos, pero tampoco debe ser con ánimo de perdida como lo explica Leal:

La RsE es algo que funciona si se aplica como debe ser. Pero, con la RsE sucede igual como cuando se impulsó el cooperativismo; es bueno en su base pero cuando quienes lo aplican lo convierten en negocio se pierde. Una cosa es que debe ser rentable y otra volverla un negocio. Muchas de las empresas que están en la Superintendencia de Sociedades y en la Bolsa de Valores lo aplican de la mano de las prácticas de buen gobierno.

Para Francisco Leal la responsabilidad social empresarial debe brindar un escenario "que contribuya a la formación en el crecimiento humano, social y económico de las personas. Crear empresas sociales que ayuden a reducir la brecha de pobreza”.

\section{La RSE brinda nuevas oportunidades}

Jacqueline Butcher de Rivas, presidenta del consejo directivo del Centro Mexicano para la Filantropía (Cemefi), afirma: "En el mundo de hoy, la responsabilidad social empresarial es una nueva forma de hacer negocios hacia la construcción del bien común".
Uno de los ejemplos más claros de diversificación de negocios a partir desde la RSE es el caso del canal internacional Discovery; con dos proyectos amplió sus alcances, bajo costos de operación en mercadeo, modernizó la interacción entre sus televidentes o usuarios e incorporó las tecnologías de la comunicación poniéndolas al servicio de la educación y el medio ambiente, todo gracias a un gran derroche de creatividad.

Sin alejarse de las pantallas, Discovery consolidó dos nuevos espacios, el primero es Discovery en la Escuela, producciones que llegan a las aulas de forma satelital o por cable, proyecto que es auspiciado por las organizaciones y personas que buscan una masificación del saber y en muchos casos es apoyado por los mismos operadores de cable, es una herramienta didáctica para los docentes que pueden preparar sus clases partiendo del menú que ofrece este proyecto, Discovery retoma sus producciones adaptándolas a un formato más sencillo para facilitar el uso del material.

El segundo es "Planeta te quiero verde", un plan focalizado al sector ambiental que involucra, desde Discovery Kids, a sus televidentes de menor edad. Fomentan que los niños y las niñas se acerquen a las nuevas tecnologías y por medio del video desarrollen propuestas que partan desde la cotidianidad para ayudar al mejoramiento del planeta. Incluyen totalmente a sus stake holders, aprovechan las actuales dinámicas tecnológicas y promueven el cuidado ambiental por medio de la formación de las nuevas generaciones; además, suscitan dinámicas familiares para la construcción de un microvideo.

Definitivamente, uno de los aspectos preponderantes en la RSE es el medio ambiente, la conciencia ecológica se debe 
incluir desde el manejo de los recursos internos de la empresa, el impacto ambiental de la producción y su aporte para la sustentabilidad de los recursos de sus entornos local, regional y mundial.

Por otra parte, los procesos formativos son muy bien recibidos y más cuando se trata de generar cimientos en los niños y las niñas, como es el caso de Faber Castell que creó la Fábrica del Ecolápiz, aprovechando que en los primeros años lo que más se hace es dibujar, colorear y la mayor distracción de los pequeños es pintar, la multinacional alemana enseña con esta atracción como se fabrican los lápices de colores utilizando un método con el cual el medio ambiente sufre un mínimo impacto.

En un acompañamiento permanente, mientras los niños oprimen infinidad de botones, van recibiendo datos curiosos como cuántos colores se obtienen de un árbol o cómo está compuesta la mina de un lápiz, durante el recorrido que dura 30 minutos y es apto para toda la familia, van apareciendo varios personajes clásicos de un castillo que les ayudan a los niños a interactuar con la Fábrica del Ecolápiz y generar un aprendizaje significativo dónde se concientiza a cerca de lo que significa un lápiz en el contexto ambiental.

Pero, no todo es color de rosa en la RSE. Si bien el panorama es prometedor al momento de pensar en negocios económicamente autosostenibles hay que tener mucho cuidado en los procesos de conformación de los mismos. El Centro de Integración, Cooperación y Desarrollo Internacional (Cicodi) que es un organismo de articulación, investigación y asesoramiento sobre temas de integración regional, cooperación y desarrollo internacional ha identificado los siguientes diez errores al momento de ejecutar proyecto de RsE:

1. Falta de diagnóstico situacional

2. Falta de planificación

3. Acciones aisladas

4. Inexperiencia en las líneas de acción

5. Escasa articulación

6. Falta de un presupuesto determinado

7. Programas inflexibles

8. Inadecuada sistematización de información

9. Falta de monitoreo del proyecto

10. Inexistencia de un plan de comunicación interno y externo.

\section{La RSE no da espera}

Al hablar con Julián Hernández Rincón connotado economista, académico y especialista en administración de la Universidad de los Andes, él ratifica la urgencia de manejar este proceso de retribución,

hoy en día, la RSE es un tema de absoluta obligatoriedad para las empresas; teniendo en cuenta que es el conjunto de acciones que deben tener un impacto en la sociedad, en el entorno de la empresa como si fuera la retribución y el reconocimiento a una sociedad que nos reconoce y acepta y nos da el estímulo a seguir haciendo las cosas como organización.

En la gran mayoría de las ocasiones los empresarios evaden la RSE argumentando la falta de recursos económicos para implementar este tipo de planes por eso

la discusión se puede determinar en razón de que aquellas empresas que tienen cierto tamaño, cierta estructura y ciertos recursos sí pueden elaborar y ejecutar proyectos de RSE; esto genera una clasificación en la que la pequeña o media empresa no se ven obligadas o no van a tener ese compromiso, cuando no necesariamente la responsabilidad debe estar asociada con tener un presupuesto o unos recursos, esto debe responder a elementos filosóficos que tenga la organización, para que se pueda devolver a un sector de la población los beneficios 
económicos que la organización ha recibido a cambio de los servicios que presta en un mercado, afirma Hernández.

Desde los hilos directivos de las empresas se debe tener en cuenta que la RsE puede generar muchas oportunidades, pero no se debe trabajar a la ligera, Julián Hernández es muy enfático frente a esto:

el compromiso de las empresas se puede desvirtuar por razones de mala interpretación o por falta de una definición más apropiada en términos de este compromiso que tienen las organizaciones. se desvirtúa por el afán que tienen las empresas para mostrar a la sociedad sus resultados dando a entender que se cumple con este compromiso. el tema que se debe revisar es si esta responsabilidad social si genera verdaderos beneficios a quienes va dirigida y no se termina en el afán de decir "lo estamos haciendo"; pero, no se está midiendo si ese compromiso se está cumpliendo.

Los procesos creativos, el conocimiento de los entornos sociales, el contar con un personal altamente proactivo y emocionalmente estable hará que se acate efectivamente el Pacto Mundial de las Naciones Unidas en materia de responsabilidad social de las empresas, idea que fue lanzada por Kofi Annan, Secretario General de la ONU, el 31 de enero de 1999 ante el World Economic Forum en Davos.

\section{Los diez principios del Pacto Mundial}

En la página www.pactomundial.org se encuentran consignados los principios que dan el marco referencial a la responsabilidad social empresarial, los cuales se centran en convenciones y declaraciones universales:

1. Las empresas deben apoyar y respetar la protección de los derechos humanos fundamentales, reconocidos internacionalmente, dentro de su ámbito de influencia.

2. Las empresas deben asegurarse de que sus empresas no son cómplices en la vulneración de los derechos humanos.
3. Las empresas deben apoyar la libertad de asociación y el reconocimiento efectivo del derecho a la negociación colectiva.

4. Las empresas deben apoyar la eliminación de toda forma de trabajo forzoso o realizado bajo coacción.

5. Las empresas deben apoyar la erradicación del trabajo infantil.

6. Las empresas deben apoyar la abolición de las prácticas de discriminación en el empleo y la ocupación.

7. Las empresas deberán mantener un enfoque preventivo que favorezca el medio ambiente.

8. Las empresas deben fomentar las iniciativas que promuevan una mayor responsabilidad ambiental.

9. Las empresas deben favorecer el desarrollo y la difusión de las tecnologías respetuosas con el medio ambiente.

10. Las empresas deben trabajar contra la corrupción en todas sus formas, incluidas extorsión y soborno.

\section{Creatividad y actualidad fórmula ideal para la RSE}

El profe Bicente (por Bicentenario) es uno de los claros ejemplos de creatividad y actualidad al momento de pensar en RSE, este caricaturesco personaje de corbatín vino tinto, vestuario en tonos tierra, bigote prominente, gafas Lennon redondas a media nariz y ojos saltones encarna a un amante de la historia del Bicentenario que pide ayuda para terminar el libro que está escribiendo sobre la navegación marítima y fluvial en el proceso de Independencia del territorio americano.

Con este pintoresco profesor la Fundación Telefónica, la Alcaldía de Cartagena de Indias y la Fundación Bicentenario conformaron Conexión Bicentenario 2010: "una aventura educativa de conocimientos marítimos e históricos a través de las nuevas tecnologías". Esta iniciativa se desarrolló en el marco del Sail Cartagena de Indias 2010, que es el festival internacional de veleros y marinos 
llegados de todo el mundo al Caribe colombiano con el propósito de conmemorar el Bicentenario del grito de Independencia.

Conexión Bicentenario 2010, visto como un proceso de RSE, parte desde la actualidad de la celebración del Bicentenario y combina las tecnologías para educar de una forma cien por ciento interactiva, fomentando una participación permanente y el espíritu de competencia entre los integrantes del programa, motivando las acciones con premios productivos que propenden por generar más procesos formativos a futuro. Además, contribuye a los procesos de construcción de redes de cooperación entre la empresa privada y los organismos de gobierno locales. Uno de los resultados más destacados es la conformación de una gran Red de Investigación del Bicentenario.

Este producto tiene como antecedente lo que fue la Conexión Picasso en el año 2008, y se retomó y se adaptó teniendo en cuenta la coyuntura del Bicentenario, esta versión se enmarca dentro del proyecto macro de la Fundación Telefónica denominado EducaRed, que como lo expresa su encargado, Juan Andrés Beltrán,

busca incorporar las nuevas tecnologías dentro de los procesos educativos a través de cualquier tipo de temática. Por ejemplo, este año estaba el tema del Bicentenario para desarrollar actividades.

Las exigencias de la construcción de todo tipo de programa de RsE, se centran en contar con un grupo conformado por diferentes profesionales que puedan aportar una visión integral, es decir primero se debe determinar, claramente, la línea de acción que se va a seguir y luego, teniendo ese punto de partida, trabajar en equipo.

En EducaRed la pluralidad profesional es amplia, somos un grupo interdisciplinario en el que se encuentran economistas, abogados, comunicadores sociales, educadores, expertos en sistemas; pero, lo que acá tenemos es una experiencia y un conocimiento de las dinámicas de las nuevas tecnologías dentro del aula y eso es lo que nos lleva a poder generar este tipo de actividades y ese tipo de iniciativas, señala Beltrán.

Los productos deben ser estudiados muy bien para que alcancen una efectividad, en la Fundación Telefónica tenemos un equipo de pedagogos, que conocen el funcionamiento de las nuevas tecnologías en el aula de clase y además de eso lo que hacemos es proponer ideas para que la dirección y gerencia de EducaRed las apruebe. Nosotros tenemos unos claros lineamientos de calidad, contamos con unas fichas de proyecto en las que podemos ver a cuántas personas le vamos a apuntar, a cuántas personas podemos beneficiar hasta llegar al producto final que es lo que a nosotros nos interesa, que no sea una actividad de un solo momento, sino que trascienda en el tiempo y que beneficie a los profesores y a los alumnos que participan en él, afirma el comunicador Juan Andrés Beltrán encargado de EducaRed.

\section{La RSE en Europa}

José Miguel Rodríguez Fernández, doctor con Premio Extraordinario en Ciencias Económicas y Empresariales por la Universidad de Valladolid y quien ha sido durante algo más de siete años decano de la Facultad de Ciencias Económicas y Empresariales de la Universidad de Valladolid y miembro de su Junta de Gobierno, accedió a contestar unas preguntas en torno a la situación de la RSE en Europa. Las experiencias del viejo continente fácilmente nos pueden servir como prospectivas para los procesos que vivimos en la región, claro está, teniendo en cuenta la globalidad de nuestra realidad y 
adaptándola totalmente al contexto latinoamericano.

\section{¿Hacia dónde se enfoca la responsabilidad social empresarial en la Unión Europea? \\ José Miguel Rodríguez Fernández}

Fundamentalmente, parece enfocarse hacia la búsqueda de buena reputación e imagen corporativa y -a través de ellas-creación de valor a largo plazo para los accionistas o propietarios, dentro de un marco donde se percibe la RSE como una opción voluntaria de las empresas, aunque con determinados impulsos desde las administraciones públicas.

\section{¿Cómo se maneja la formación de los estudiantes universitarios frente al tema de RSE? JMRF}

En diversos países las administraciones públicas responsables de las universidades, las agencias de acreditación de la calidad de las universidades, o las propias universidades han aprovechado las recientes reformas de las titulaciones y de los planes de estudios universitarios para incluir la formación en RSE como una competencia o habilidad que debe haberse alcanzado al terminar los correspondientes estudios. Pero las vías para conseguirlo están siendo diversas. En algunos casos, existen asignaturas específicas sobre esa materia (unas veces son asignaturas obligatorias y otras veces son optativas). En otras ocasiones, se espera que el tema de la RSE sea abordado de forma transversal en varias asignaturas simultáneamente, cada una desde su perspectiva. Desde el punto de vista didáctico, la formación teórica en la materia puede completarse con el análisis de casos, realización de visitas o estancias de estudiantes en empresas que tienen políticas de RSE, etcétera. Aparte de todo esto, empiezan a abundar los títulos de posgrado dedicados en exclusiva a la formación en RSE o sostenibilidad.

\section{¿Cómo se puede manejar la RSE, para que tenga un impacto global y no solo un impacto local? JMRF}

Me parece que ante todo es necesario ponerse mínimamente de acuerdo sobre lo qué es la RSE. Hoy por hoy, es un concepto maleable, casi libremente interpretable, difuso y, al final, confuso. En parte, por ello se generan las incoherencias entre lo que se observa en el ámbito local y lo que se percibe en un contexto global.

Empresas claramente multinacionales, o simplemente grandes pero operando en varios países, tienen comportamientos más responsables en algunos lugares (por lo general, en sus propios países de origen del Norte desarrollado) que en las naciones del Sur en desarrollo. Detrás de ello no solo hay una incoherencia evidente, sino también una concepción relativista de la RSE: se tiene más cuidado en el Norte porque se cree que puede difundirse más rápidamente la información acerca de una posible conducta irresponsable o puede afectar más a su imagen justo donde radican sus principales o más influyentes inversores, clientes, etcétera.

Una ventaja de la globalización de las informaciones y la progresiva mayor facilidad de acceso de diversas poblaciones a internet es que este tipo de razonamiento empieza a ser poco apropiado. Cuando por fin se llegase a mantener comportamientos coherentes en todos los países de actuación, habría de evidenciarse un mismo respeto a los derechos humanos -individuales
Fundamentalmente, parece enfocarse hacia la búsqueda de buena reputación e imagen corporativa $y$-a través de ellas-creación de valor a largo plazo para los accionistaso propietarios, dentro de un marco donde se percibe la RSE como una opción voluntaria de las empresas, aunque con determinados impulsos desde las administraciones públicas. 
y colectivos o comunitarios-; una retribución por hora similar en términos reales (es decir, en términos de poder adquisitivo) para los trabajadores de la empresa que realicen análogas actividades en los diversos países; una misma preocupación en cuanto a los riesgos laborales, la preservación del medio ambiente o la protección del cliente o consumidor; un control similar de la responsabilidad social de los diversos proveedores incluidos en la cadena de suministros.

Para llegar a todo ello, además de la difusión y el contraste de informaciones, parece importante la acción de los medios de comunicación independientes y de las organizaciones no gubernamentales (ONG). Pero también cabe preguntarse si no sería conveniente una cierta regulación o reglamentación de la RSE en el plano mundial, elaborando esa regulación dentro de algún organismo político o económico internacional (ONU, OCDE, UNCTAD, etcétera).

La actual crisis económica y financiera internacional ha puesto una vez más de manifiesto que no puede dejarse la RSE en manos de la mera voluntad de las empresas (la irresponsabilidad de las empresas financieras ha sido evidente), aunque solo sea porque los mismos defensores del mercado sin control y la autorregulación suelen partir justamente de un modelo de racionalidad humana en que solo el interés personal guía el comportamiento. Y llevan más de dos siglos sin poder demostrar en la práctica, de forma rigurosa y sólida, que el egoísmo personal conduce-por medio de no se sabe qué vía milagrosas-al bienestar colectivo.

\section{¿Cómo debe manejar una empresa los incrementos económicos que implica el establecimiento de programas de Responsabilidad Social Empresarial?}

Parece cierto que la mayor parte de los enfoques sobre RSE manejados en las empresas se basan en un concepto de RSE que las lleva a hablar de inversión en RSE y consiguiente logro de resultados, en último término resultados económicos. Los directivos o gerentes más favorables hacia las políticas de RsE hablan de que es una inversión y no un gasto.

Reconozco que no me gusta ese tipo de lenguaje, aunque sin duda represente un avance frente a otras opiniones más contrarias a la RSE. Este lenguaje pone en evidencia un análisis de la RSE análogo al aplicado para evaluar inversiones en máquinas o en publicidad y relaciones públicas ¿Quiere decir que, entonces, se abandonarían las políticas de RSE en cuanto los encargados en la empresa de la contabilidad y las finanzas pusieran encima del escritorio previsiones o pronósticos que demostrasen que, incluso a largo plazo, una acción concreta de RSE no se espera que genere un incremento de la riqueza de los accionistas o propietarios? Por tanto, el primer punto a tener en cuenta a la hora de manejar el coste de los programas de RsE es que no hay que manejarlos como costes, ni siquiera como inversiones. Los programas de RSE son un imperativo moral, derivado de fundamentaciones éticas y de justicia que obligan a las empresas tanto como a las personas individuales. A la vez, esos programas de RSE tienen también una fundamentación de análisis económico, ¡pero que no es un análisis de rentabilidad!

Con esto último lo que quiero decir es que el análisis o la teoría económica siempre han advertido de que es necesario "internalizar" -es decir, tener en cuenta y computar-los efectos externos ("externalidades") 
derivados de la actuación de los diferentes actores sociales, por ejemplo, las empresas. Del mismo modo que se dice "quien contamina, paga", también habría que decir "quien destruye injustificadamente puestos de trabajo, reduce el bienestar social de una comunidad local o proporciona un mala calidad en sus productos, paga”. Además, existe una vertiente en que se combina la ética con esta visión del análisis económico. Y es que, además de todo lo anterior, la creación de valor para un grupo (por ejemplo, los accionistas) no puede hacerse a costa de otro (por ejemplo, los clientes), tanto por razones morales como porque eso supondría una expropiación o expolio de rentas por los primeros a expensas de los segundos, quienes abandonarán la empresa. Más en general, no hay razones para que haya de crearse valor para un grupo y no también para otros grupos.

\section{Si bien la RSE es un proceso voluntario ¿Cuáles son los puntos preponderantes para que una empresa se motive a desarrollar un proceso de RSE?}

Hoy por hoy, da la impresión de que embarcarse en un proceso de RSE suele ser la consecuencia lógica de varias razones que se entremezclan: el tema está de moda y la propia presión social y mediática obliga a no situarse al margen de él; los expertos en mercadotecnia y reputación corporativa no dudan de sus diversas ventajas para la imagen de la empresa; los gerentes de finanzas se están convenciendo de que el valor de las acciones puede aumentar a largo plazo; el director de recursos humanos vislumbra que la RSE puede atraer a mejores especialistas como empleados de la organización $y$, a la vez, motivar y unir a la plantilla de personal; la alta dirección tiende a entender que la RSE puede ser una excelente vía para ganar legitimidad social, e incluso mejorar la posición de la empresa ante los gobiernos y las administraciones públicas, etcétera. Además, todos los responsables de introducir y aplicar políticas o acciones de RSE pueden sentirse mejor desde su vertiente afectiva y emocional.

\section{¿Cuáles son los obstáculos más recurrentes al momento de desarrollar programas de responsabilidad social empresarial en Europa?}

Ante todo, la propia diversidad conceptual y de los programas sobre RsE. Esa diversidad implica en la práctica incurrir a veces en contradicciones evidentes; presentar memorias o informes de RSE, que destacan los logros socialmente más apreciables pero nada dicen sobre los "puntos oscuros" de las propias acciones empresariales. ¿No habría de avanzarse hacia una RSE con menos discurso simbólico, menos cosmética, y mucho más contenido real, incluso en términos de más justa distribución de la renta y la riqueza?, ¿no habría de reenfocarse la formación de directivos y gerentes en las escuelas de negocios, para que perciban la RSE como un compromiso moral y una obligación económica de crear valor de forma equilibrada para todas y cada una de las partes interesadas o stake holders, no solo para los accionistas?, ¿ de verdad es creíble un programa de RSE en, por ejemplo, un gran banco que haya contribuido a la actual crisis financiera, haya recibido ayuda de fondos públicos y ahora utilice su dinero para especular en los mercados de bonos gubernamentales, resistirse ante mayores regulaciones o presionar para que los gobiernos no limiten las desorbitadas retribuciones de sus directivos? 
Por otra parte, ¿realmente se puede dejar solo en manos de las empresas aplicar de forma voluntaria políticas de RSE?, ¿no tienen nada que decir los gobiernos y administraciones públicas?, ¿no sería conveniente un determinado conjunto de regulaciones que introdujeran algunas exigencias en el ámbito de la RSE, además de animar a la aplicación voluntaria de políticas al respecto? Al final, la pregunta puede ser: ¿cabe una auténtica RSE en el actual modelo económico y social sin introducir reformas de calado, que impliquen una reestructuración de las bases ideológicas, económicas y políticas, sobre las que se ha edificado el orden mundial durante los últimos treinta años y que tan tranquila y estupendamente están resistiendo las presiones de la opinión pública para introducir cambios?

La responsabilidad social empresarial es una necesidad para Colombia, sin excluir ningún tipo de organización, y los programas que se implementen deben estar elaborados bajo una línea clara y específica que se base en el contexto de nuestra realidad y la creatividad de un grupo interdisciplinar que optimice todos los recursos posibles y en lo posible recordando las palabras del escritor y periodista uruguayo Eduardo Galeano " $\mathrm{La}$ caridad es humillante porque se ejerce verticalmente y desde arriba; la solidaridad es horizontal e implica respeto mutuo".

\section{Bibliografía}

1. CCRE, Centro Colombiano de Responsabilidad Empresarial. Recuperado el 19 de abril de 2010 de http://www.ccre.org.co

2. Fernández García, Ricardo. (2008). Responsabilidad social corporativa. España: Editorial Club Universitario.

3. Ramírez Padilla, David Noel. (2007). Integridad en las empresas, ética para los nuevos tiempos. México: McGraw Hill.

4. Werther, William B. Jr.; Chandler David. (2010). Strategic Corporate Social Responsibility, Stakeholbal in a Global Environmente. United States of America: SAGE Publications, Inc. (segunda edición).

5. Unión Europea. (2001). El libro verde para fomentar la responsabilidad social de las empresas. Bruselas.

6. Fernández, Ana; Gutiérrez, Evaristo. (2010). Responsabilidad social empresarial: modelos de aplicación. En Forum calidad, año 21 (209), p. 62-68.

7. Universidad Antonio Nariño. (2010). Revista Enlace Empresarial, Edición 23 Año 2010. Colombia. Disponible en: http://www.revistaenlaceuan.com/edicion23/ inicio.html

8. RS Revista, Publicación de Gatos Gemelos. (2011). Colombia. Disponible en: http:// www.rsrevista.com

\section{Páginas web}

- http://www.pactomundial.org

- http://www.alternativaresponsable.org

- http://www.colombiaincluyente.org 